
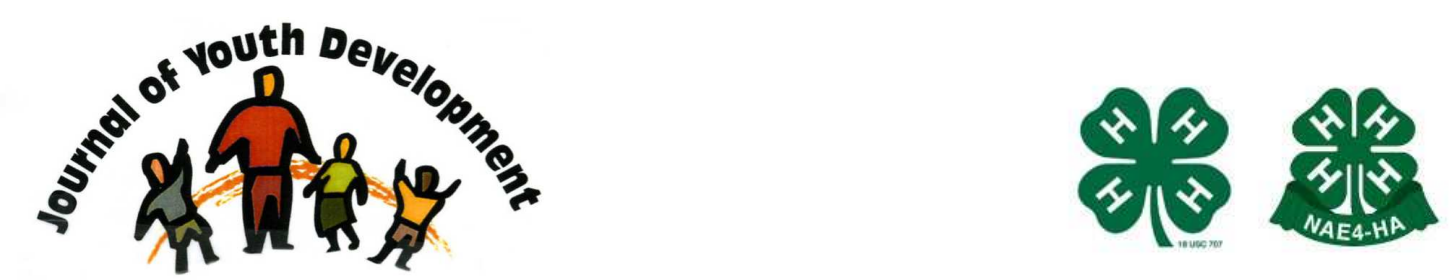

Bridging Research \& Practice

\title{
Where PYD Meets CBPR: A Photovoice Program for Latino Immigrant Youth
}

\author{
Elizabeth Andrade \\ Milken Institute School of Public Health \\ The George Washington University \\ Washington, DC \\ elandrade@gwu.edu \\ I.C. Cubilla \\ The George Washington University \\ Washington, DC \\ G. Sojo-Lara \\ The George Washington University \\ Washington, DC \\ S.D. Cleary \\ The George Washington University \\ Washington, DC \\ M.C. Edberg \\ The George Washington University \\ Washington, DC \\ L.K. Simmons \\ The George Washington University \\ Washington, DC
}




\title{
JOURNAL OF YOUTH DEVELOPMENT \\ bridging research and practice

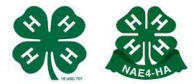

Volume 10, Number 2, Summer 2015

Article 151002FA003

\section{Where PYD Meets CBPR: A Photovoice Program for Latino Immigrant Youth}

\author{
Elizabeth Andrade, E.L. Andrade, I.C. Cubilla, G. Sojo-Lara, S.D. Cleary, \\ M.C. Edberg and L.K. Simmons \\ The George Washington University
}

\begin{abstract}
Community engagement in identifying issues of collective concern to address health disparities is an approach that is central to conducting community-based participatory research. It is particularly important for youth to be engaged in dialogue around issues that affect their lives. Participation of this nature is understood, within a Positive Youth Development (PYD) approach, to be an element of primary prevention vis a vis health risks. Photovoice has been an increasingly used methodology to enable youth to identify and address issues relevant to their daily experiences. We implemented a six-week Photovoice project guided by a PYD approach with Latino immigrant youth $(n=12)$ from Langley Park, MD. This article describes the experiences of facilitators in implementing the program, testing a new curriculum, and also presents results related to changes in PYD assets among participants. We also offer recommendations for future Photovoice programs with similar populations and aims.
\end{abstract}

\section{Introduction}

Latinos are the largest ethnic minority group in the U.S., accounting for $16.9 \%$ of the total population (US Census Bureau, 2012), thus a key population of focus with respect to addressing health disparities. Over the past few decades, the Washington, DC area has been a major destination for a number of immigrant groups, particularly from Central and South American countries. According to the U.S. Census, between 2000 and 2010, the state of Maryland experienced a $107 \%$ increase in Latino residents. The community of Langley Park, MD is a burgeoning immigrant community outside of Washington, DC (pop. 20,675). Langley Park is predominantly low-income and foreign-born (67.6\%), with $79.7 \%$ of residents self-reporting as Latino (US Census Bureau, 2012). Data collected in a recent study by Cleary et al. showed the national origins of the foreign-born Latino population in Langley Park as primarily from El 
Salvador (46.53\%), Guatemala (32.86\%), and Honduras (10.41\%). The same study estimates that among foreign-born Latinos ages $12-17,66.33 \%$ have lived in the U.S. three years or less (Cleary, 2014), which is consistent with a recent wave of unaccompanied minor immigrants from Central America choosing Langley Park as their final destination. Latino youth, including those living in Langley Park, experience numerous health disparities and elevated risk behaviors, one of which is the co-occurrence of substance use, sexual risk, and interpersonal violence (Edberg, et al., 2009; Martinez Jr., 2006; Martinez, Eddy, \& DeGarmo, 2003; Vega \& Gil, 1998). We implemented a six-week Photovoice project guided by a Positive Youth Development (PYD) approach with Latino immigrant youth from Langley Park, MD. This article describes the experiences of facilitators in implementing the program, testing a new curriculum, and also presents results related to changes in PYD assets among youth participants.

The Avance Center for the Advancement of Immigrant/Refugee Health (Avance Center) formed a collaborative partnership with the community of Langley Park, MD to address health disparities experienced by Latino immigrant youth and their families. Central to the work of the Avance Center is the Adelante intervention, a community-based, multi-level PYD program that addresses the co-occurrence of substance abuse, sexual risk and interpersonal violence among Latino youth in Langley Park. The Avance Center's key implementation partner is the Maryland Multicultural Youth Center (MMYC). The overarching goal of Adelante is to build individual, family and community assets -- Competence, Connection, Confidence, Contribution representing 4 of 6 key PYD constructs, referred to as " $C$ 's" (Lerner, 2005; Silbereisen, \& Lerner, 2007), and connections between these assets, as a mechanism for preventing youth risk behavior. Recognizing the social-ecological nature of health problems, the Adelante intervention is innovative, community-driven, and addresses multiple contributing factors for these health disparities. The Photovoice pilot program described here is part of the Adelante intervention, and is also part of a larger university-community participatory research process. In this article, we describe the development and a testing a Photovoice curriculum with immigrant, Latino youth participants living in Langley Park, as well as the impact of the program on changes in PYD assets among participants. We also offer recommendations for future Photovoice programs with similar populations and aims.

\section{Background}

Community engagement in identifying and prioritizing issues of collective concern to address health disparities is an approach that is central to conducting community-based participatory research (CBPR) (Israel, Eng, Schulz, \& Parker, 2005; Minkler, \& Wallerstein, 2003). CBPR is designed to build capacity among community members and stakeholders to engage in research and address community issues (Agency for Healthcare Research and Quality, 2009). It is important for marginalized and vulnerable groups to have a voice through this process, but it is not always the case that these groups are empowered to speak out. Immigrants, in general, and immigrant Latino youth, in particular, tend to be at increased risk for exclusion from these community participatory processes (Roffman, Suarez-Orozco, \& Rhodes, 2003). Moreover, it is particularly important for youth to be engaged in dialogue around issues that affect their lives and to be empowered to take action if barriers and injustices are identified. Participation of this nature is understood, within a PYD approach, to be an element of primary prevention vis a vis health risks. Active participation during adolescence can also contribute to more sustained prevention effects, because it occurs during the formation of a social identity, a key adolescent developmental process (Erikson, 1968). To support this development, adolescents should be given the tools and opportunity to develop their communication skills, share their insights, and become agents of change (Youniss, \& Yates, 1997). The Photovoice process is an excellent strategy for achieving this goal, allowing young people an opportunity to explore different social 
phenomena, culture, and behaviors, thus enhancing their understanding of themselves and world they live in (Strack, Magill, \& McDonagh, 2004).

In addition, photography has increasingly been used as a qualitative method for conducting participatory action research and engaging communities in health promotion and social change (Hergenrather, Rhodes, Cowan, Bardhoshi, \& Pula, 2009). Photovoice is an innovative methodology in which community members are asked to identify issues relevant to their daily experiences, portray them through photographs, reflect on the meaning and themes underlying these images, and disseminate photographic representations to policymakers with the goal of addressing disparities or inequities (Killion, \& Wang, 2000; Wang, \& Burris, 1997; Wang, Morrel-Samuels, Hutchison, Bell, \& Pestronk, 2004). Photovoice draws from Paulo Freire's approach to education for critical consciousness (Freire, 1973), positing that critical reflection leads to discovery and action to address social inequalities. The Photovoice methodology has been successfully used with different marginalized immigrant and youth populations for the conduct of CBPR (Garcia, et al., 2013; Greene, Burke, \& McKenna, 2013; Morales-Campos, Parra-Medina, \& Esparza, 2015; Rhodes, et al., 2009; Schwartz, Sable, Dannerbeck, \& Campbell, 2007; Stevens, 2010; Strack, et al., 2004; Streng, et al., 2004; Vaughn, L.M., RojasGuyler, \& Howell, 2008).

This methodology is especially useful for engaging immigrant youth because they may be unfamiliar with processes of meaningful community change where their voices count (Greene, et al., 2013), they may experience barriers to expressing their opinions, or they may feel disempowered because of their immigration experiences or status (Streng, et al., 2004). This is particularly relevant for the scores of recently arrived Latino immigrant youth in the U.S., an especially vulnerable population. Despite the use of Photovoice with U.S.-born and more recently arrived immigrant Latino youth, none of these studies have expressly mentioned the use of a PYD-based Photovoice curricula that has been tested with a mixed youth population (both recently arrived and first generation immigrant) and adapted to meet the unique circumstances of this group of youth (Hergenrather, et al., 2009; Morales-Campos, et al., 2015; Streng, et al., 2004; S. Vaughn, 2012). The reality is that many immigrant enclave communities are increasingly composed of youth with varying lengths of time living in the U.S. and varying degrees of engagement in community issues. Having a curriculum that is tailored to meet the needs of this diverse population is a useful tool to facilitate more effective engagement of Latino immigrant youth in participatory action research.

\section{Methods}

As part of the overall CBPR and prevention strategies, the Adelante project implemented a Photovoice pilot program with Latino immigrant youth living in Langley Park, MD. This program was a collaboration between youth participants and university researchers, (Wang, \& Burris, 1997) and served as both a participatory needs assessment to inform investigators, as well as an empowering, skill-building activity for youth. The Photovoice program contributed to the goals of the overall Adelante intervention, and introduced the Latino youth viewpoint into a broader dialogue around community needs, where this voice was previously unheard. The specific aims of the Photovoice program were to: update the research team about evolving community needs/strengths from a youth perspective in order to inform the ongoing Adelante intervention; develop youth skills in photography, community activism, and leadership; build PYD assets among participants regarding the constructs of Confidence, Connection, and Competence; engage youth in a dialogue around community issues; and empower youth to advocate for change to local officials to address identified disparities. 


\section{Overview of Photovoice Program}

With respect to PYD aims, the Photovoice program was designed to develop youths' Confidence through learning new skills like photography and public speaking, while also building a deeper Connection to their community, their peers and their cultural heritage. The Photovoice program was also intended to enhance participants' Competence to take action related to community issues that were important to them. From a social-ecological perspective, this program sought to not only build youths' individual assets, but to connect them to their broader community context in a dynamic process of change.

The program consisted of six weekly sessions that were conducted after school, followed by three photo exhibitions and an online exhibition. Youth were provided digital cameras and week-long photo assignments to answer the following questions:

1) What is it like being a youth living in your community?

2) What do you like/dislike about your community?

3) What are things that help you/prevent you from being healthy?

Participants were also encouraged to take photos that captured their thoughts, feelings, or artistic perspective. The overarching purpose of the photo assignments was to capture community needs and strengths from a youth perspective. Each photovoice session generally followed a process: review/discussion of photos from that week's photo assignment, a presentation/discussion about community issues or photography-specific topics, and introduction of the new photo assignments and brainstorming on the types of issues that might be portrayed through photos for that assignment. Sessions lasted two hours, and were longer for field trips.

The SHOWeD method (Wang, et al., 2004) was used for the review and analysis of photos taken by youth, with additional probing questions used when necessary. This method included five questions:

1) What do you see here?

2) What is really happening here?

3) How does this relate to your lives?

4) Why does this situation, concern, or strength exist?

5) What can we do about it?

Photos were displayed using a projector, and we viewed all photos taken as a group. Youth selected five photos each to discuss in depth. We documented exhibition candidates based on photo quality, composition and content. Details of findings related to the community issues identified are discussed in a forthcoming article.

\section{Curriculum Development and Testing}

The research team developed a six-session curriculum to test with immigrant Latino youth having different characteristics in terms of time living in the US, age, primary language, level of engagement in community issues, and prior Adelante participation. We were interested to see how youth with different characteristics responded to and benefitted from the curriculum. Based on our experiences, we added one additional session and three optional modules:

1) Team-building and Self-Empowerment,

2) Community Activism and

3) Transitioning to the U.S, which are discussed further below. 
As noted, the curriculum was also developed to build PYD assets that had been operationalized for immigrant Latino youth as part of the larger Adelante intervention. Photovoice sessions were created for in-school youth who lived within a defined community boundary. We designed sessions to stimulate discussion and critical thinking about community, family, cultural, and immigration-related factors, as well as how their lives were influenced by these factors. Youth were encouraged to consider ways of documenting these issues through photography, expressing their opinions, and making recommendations on how to address issues that were important to them.

\section{Setting and Participants}

Participants were all Latino youth ages 12-16 living in the community of Langley Park, MD. There were 12 participants, with eight females and four males. Six youth were U.S.-born and six were born in El Salvador or Guatemala. For foreign-born youth, three had arrived in the U.S. less than three years prior, and three had lived in the U.S. for more than three years. More recently arrived youth were predominantly Spanish-speaking, and others were bilingual in Spanish and English. All participants were enrolled in public middle or high school, and were recruited through verbal invitation at Adelante youth program activities, at the high school, and through referrals. We intentionally recruited youth participants who varied in terms of length of time living in the U.S., previous Adelante program involvement, age and predominant language in order to test the curriculum and program with youth having different characteristics.

\section{Photo Exhibits}

Three in-person and one online photo exhibits were held to display the youth's artistic point of view, raise awareness about community issues, and advocate for solutions. The first exhibit took place at the Adelante program's leadership program graduation, reaching a broader audience of parents, family members, youth leaders, and community stakeholders. The second exhibit was at a monthly county-supported, community stakeholder group meeting, called the Transforming Neighborhoods Initiative (TNI), where youth presented three issues that they proposed to address in collaboration with stakeholders. The third exhibit was at a Latino health disparities conference attended by academicians, researchers, and community service providers. We are displaying the online exhibit through the Adelante program's website, http://www.adelantelp.org/.

\section{Facilitators}

Facilitators were all from The George Washington University, and included: one Assistant Research Professor, one Doctoral student, one undergraduate student and one research staff. Two facilitators were Latinas and two were non-Latinas. All facilitators were fully bilingual and had extensive experience working with Latino immigrant youth. Facilitators were familiar with some of the Photovoice youth, which created an enhanced sense of trust. Two of the facilitators had photography training.

\section{Data Collection and Measures}

Qualitative data collection included the collection of photos taken by youth, discussions of these photos, and notes taken by facilitators. Although we briefly mention the results of the Photovoice study here, detailed results are presented elsewhere. This article focuses on the experiences of facilitators in implementing the program and changes in PYD assets among youth participants. Pre-post tests were given to all participants and measured the following PYD constructs, as operationalized by the larger Adelante intervention study (See Table 1): Confidence, Connection to Community, Connection to Peers, Connection to Culture/Identity, and Competence for Civic Action. 
Table 1

Adelante Intervention PYD Construct Definitions

\begin{tabular}{|l|l|}
\hline \multicolumn{1}{|c|}{ PYD Construct } & \multicolumn{1}{c|}{ Definition } \\
\hline Confidence & $\begin{array}{l}\text { Components that increase positive self-image, such as a cultural } \\
\text { activity celebrating Latinos in the U.S.; or 2) components that } \\
\text { increase confidence in taking a specific action - role-play } \\
\text { exercises that increase youths' self-esteem or confidence that } \\
\text { they can communicate effectively to a potential employer, } \\
\text { negotiate condom use with a partner or express their opinion on } \\
\text { a topic. }\end{array}$ \\
\hline $\begin{array}{l}\text { Connection to } \\
\text { Community }\end{array}$ & $\begin{array}{l}\text { 1) Components that promote a positive feeling about or bonding } \\
\text { to the community, increase the perceived value of community } \\
\text { engagement; or 2) activities that create actual relationships to the } \\
\text { community, such as mentoring, connecting with community } \\
\text { businesses, community journalism/blogging. }\end{array}$ \\
\hline Connection to Peers & $\begin{array}{l}\text { Components that promote the value of supportive peers/friends, } \\
\text { of involvement with peers/friends who are heading in a positive } \\
\text { direction; or 2) components that actually provide activities or } \\
\text { opportunities to promote peer relationships, e.g., through group } \\
\text { activities, outings. }\end{array}$ \\
\hline $\begin{array}{l}\text { Connection to } \\
\text { Culture/Identity }\end{array}$ & $\begin{array}{l}\text { Components that promote a positive connection to cultural and } \\
\text { social heritage, such as museum trips, films about immigration, } \\
\text { talks by successful Latinos, participation in Hispanic Heritage } \\
\text { month events, etc. }\end{array}$ \\
\hline $\begin{array}{l}\text { Competence for Civic } \\
\text { Action }\end{array}$ & $\begin{array}{l}\text { Components that increase knowledge or skills about how to } \\
\text { engage in civic action, including advocacy skills, how to organize } \\
\text { a petition, or public communication skills. }\end{array}$ \\
\hline
\end{tabular}

Pre-post tests included five subscales, one for each PYD construct evaluated, each with 5 or six items rated on a 5-point Likert scale from strongly disagree to strongly agree. Since this was a pilot program, sample size was reduced, and basic analysis was done only for curriculum evaluation purposes. Participants were classified into groups for the analysis, based on key features of our study population: place of birth (U.S. vs. foreign-born), and previous Adelante program participation (previous Adelante participation vs. none). Pre- and post-test results for participants were evaluated to verify increases, decreases or no variation in PYD assets.

\section{Results}

Overall, the Photovoice program showed a great deal of promise as a mechanism for youth expression, as well as awareness-raising and advocacy on important community issues. The curriculum and SHOWeD method were useful in working with this group of youth, with a few exceptions, described below. Similar to the participatory planning process used by MoralesCampos (2015), the Photovoice project also provided an important mechanism for youth to be involved in the future direction and planning of the Adelante intervention by identifying community issues to be addressed by the program. For intervention purposes, as a strategy, Photovoice also produced gains in PYD assets (see Figures 1-3). Our experiences implementing the curriculum indicate that the Photovoice program also has potential to build skills in the 
areas of leadership, advocacy, communication and public speaking, artistic expression, and photography, although these were not directly measured.

Photographs were useful tools for youth to express their opinions, ideas and concerns. Community issues identified by youth participants through photography focused on community needs (trash removal, affordable adolescent recreation/physical activity opportunities, enhanced community safety) and community assets (culture and traditions, religion, strong family ties, availability of green spaces). Identified issues also highlighted major health threats, including high levels of substance and alcohol abuse in the community, overabundance of fast food outlets, and the high cost of fresh produce. Finally, youth expressed some troubling sentiments, including feelings of isolation, sadness and challenges that arose from their experiences as immigrants. Details on themes that emerged from the Photovoice program are presented elsewhere.

\section{Changes in PYD Constructs}

As part of the larger Adelante PYD intervention, this curriculum helped to enhance assets of youth in the areas of Confidence, Connection to Peers, Connection to Community, Connection to Culture/Identity, and Competence for Civic Action (See Figure 1), with important distinctions by place of birth and previous membership to the Adelante program, described below. Half of participants showed increases in Confidence, Connection to Peers, Connection to Community, and two-thirds of participants showed increases in Competence for Civic Action. Less improvement was seen for Connection to Culture/Identity, but still four out of twelve participants improved.

Figure 1

Percent of Overall Participants with Variation in Pre/Post PYD Asset

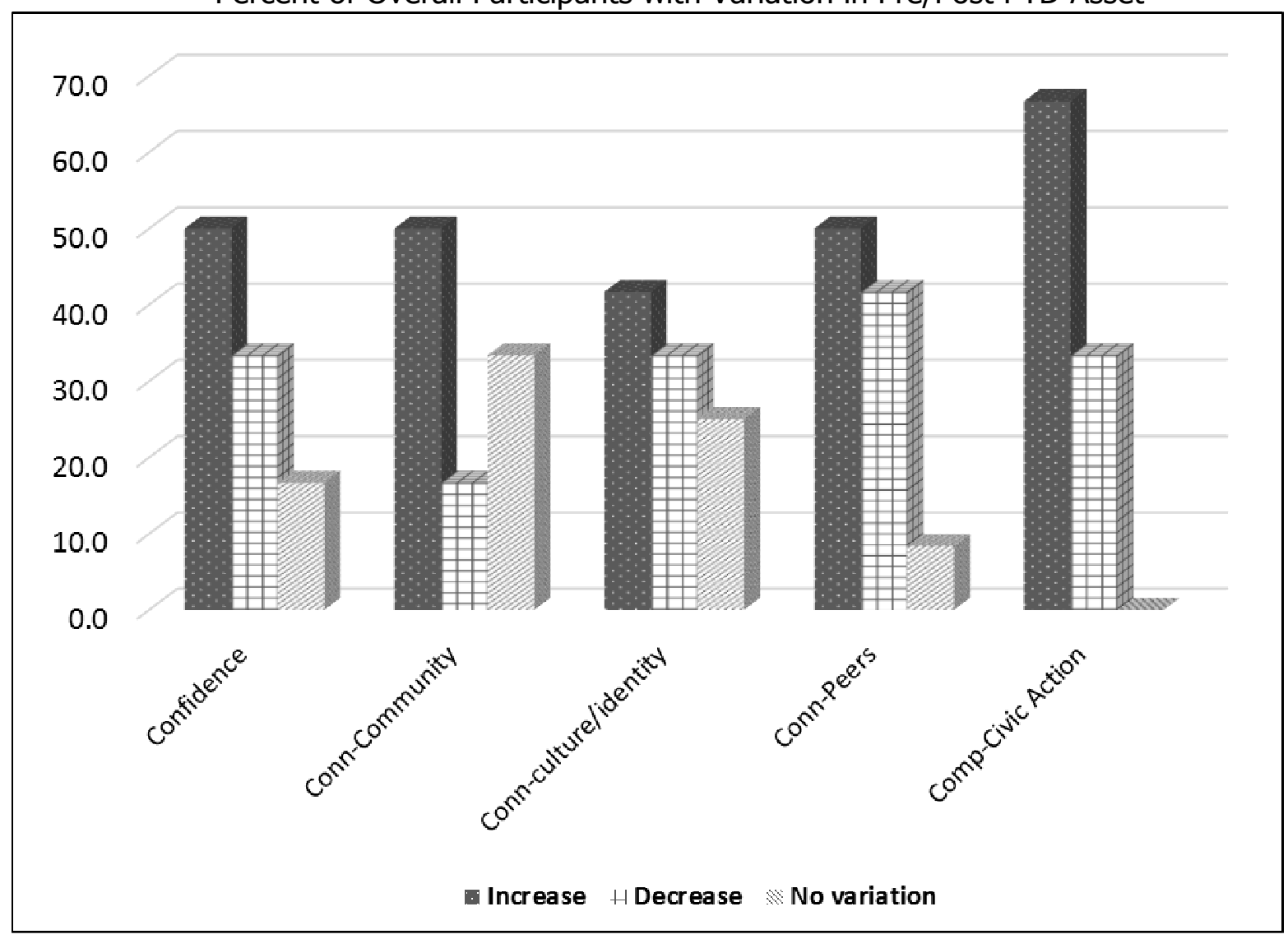


The results of the pre-post test for Photovoice participants were also analyzed from two important perspectives. The first looked at results by place of birth, in the U.S. or in Central America (See Figure 2). U.S.-born youth tended to benefit most from the program, with more of them showing increases in Confidence (67\%), Connection to Community (67\%), Connection to Culture/Identity (50\%), and Civic Action Competence (50\%). Foreign-born youth showed considerable increases in Civic Action Competence ( $82 \%$ of foreign-born participants) as a result of participating in the program and half of both U.S.-born and foreign-born youth showed increases in Connection to Peers.

Figure 2

Percent of Participants with Variation in Pre/Post PYD Assets by Place of Birth

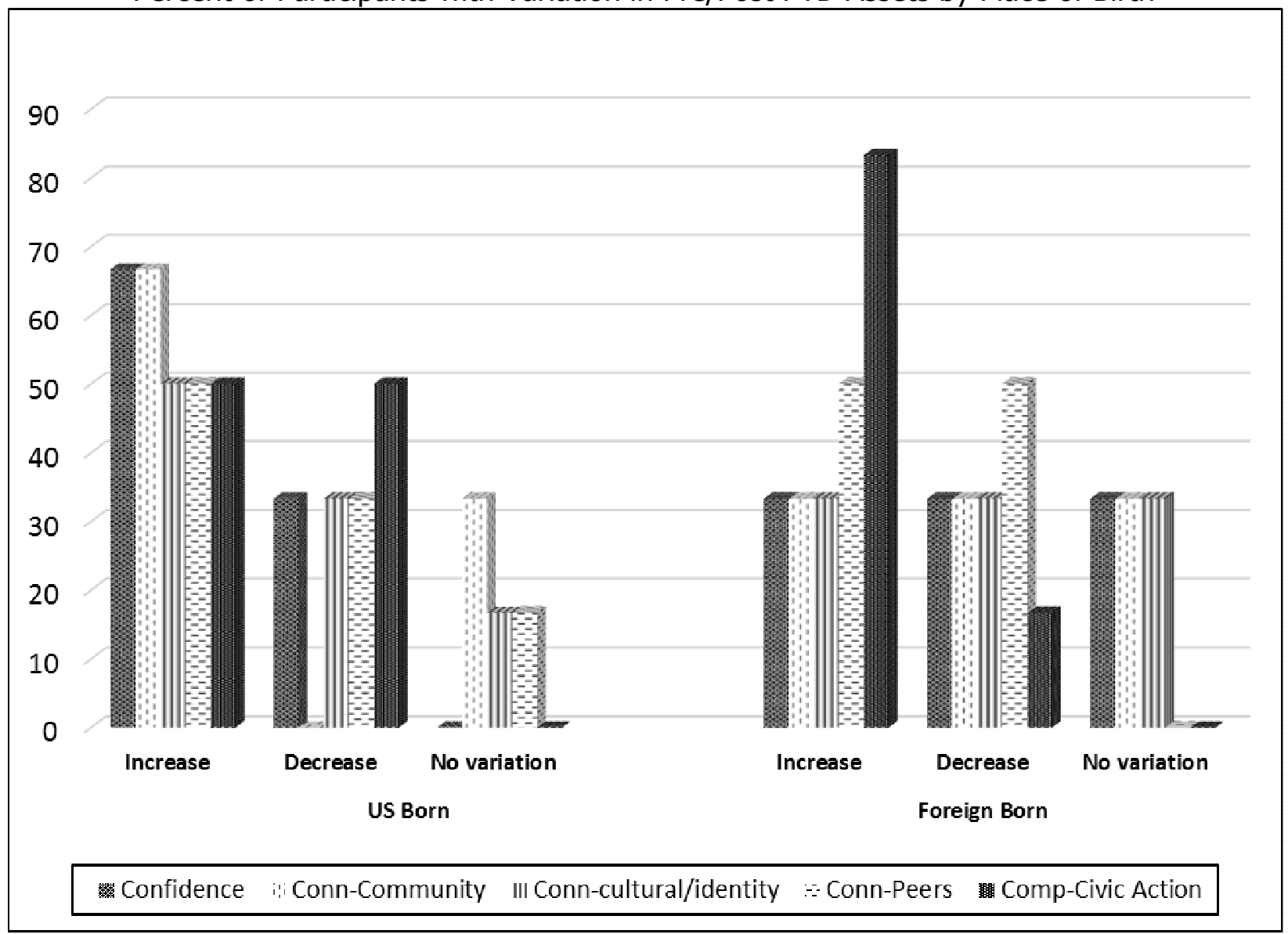

The second perspective was to look at results by whether the youth were new to the Adelante program or had participated in other Adelanterelated program activities before, since these activities are also designed to build PYD assets and we considered them to be activities that could potentially prime participants for the Photovoice experience (See Figure 3). More youth who were previous Adelante program participants showed increases in Confidence (67\% vs. $33 \%)$, Connection to Culture/Identity (67\% vs. $17 \%$ ), and Connection to Peers ( $83 \%$ vs. $17 \%)$. Both participants who were new to Adelante and those who had previous Adelante experience showed increases in Connection to Community (50\%) as a result of participation in Photovoice, as well as Competence for Civic Action (67\%). 
Figure 3

Percent of Participants with Variation in Pre/Post PYD Assets by Adelante Membership

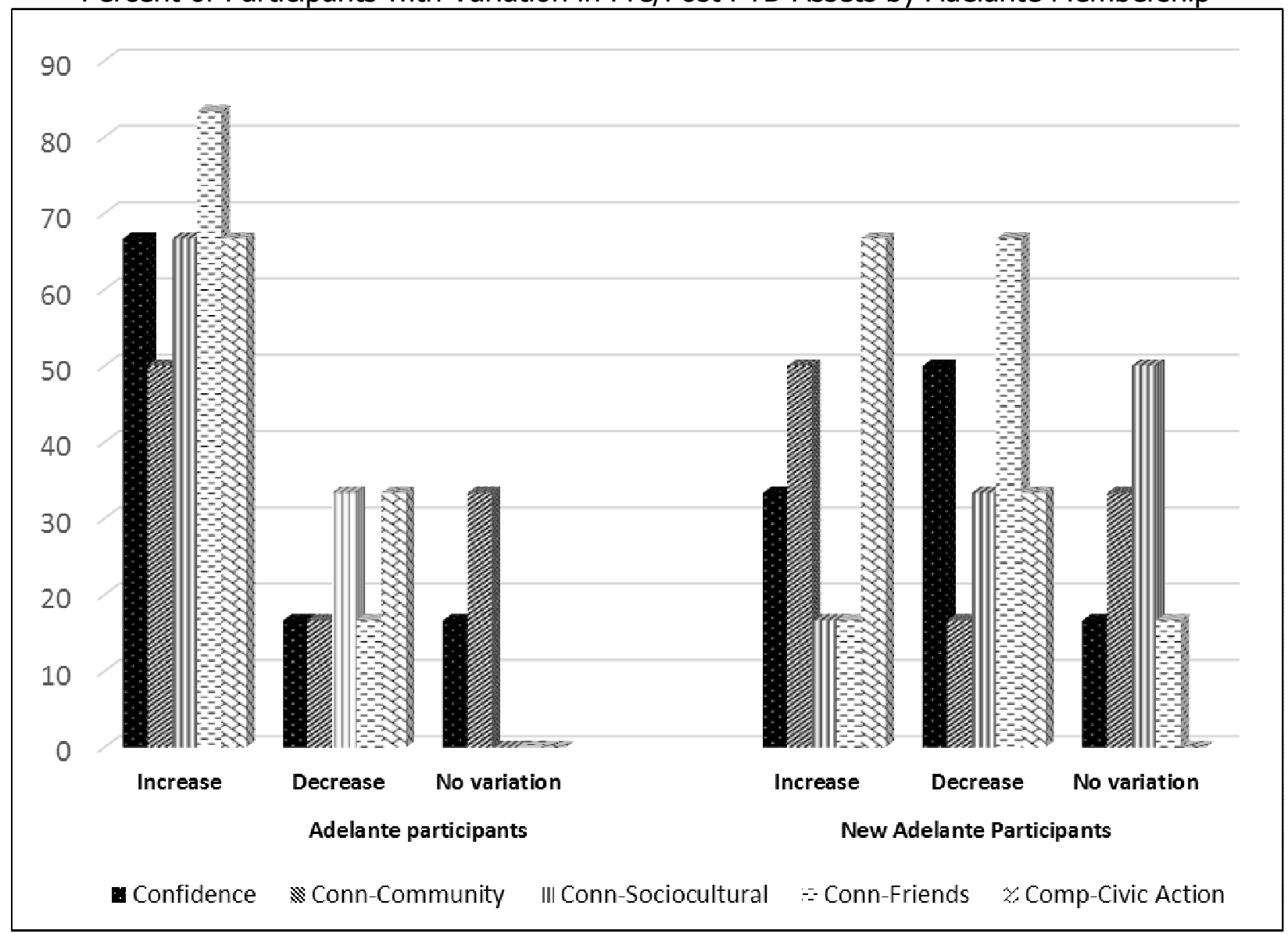

\section{Testing of Curriculum}

The curriculum was useful in working with immigrant Latino youth with diverse characteristics, such as length of time living in the U.S., previous participation in the Adelante program, age and preferred language. Described below are some salient experiences from working with this group of youth, and recommendations for future programs, including a modified curriculum (See Figure 4) with optional modules based on participant needs and characteristics.

\section{Level of Comfort with Expressing Ideas}

Participants in the Photovoice pilot program differed in their level of comfort expressing themselves in front of a group. Those participants who were seemingly less comfortable voicing their opinions were typically younger, had recently immigrated to the U.S., or had never participated in an Adelante program activity before. Participants with these characteristics were more hesitant in expressing themselves verbally, in front of a group, or through artistic expression. After participating in the program, more than half of U.S.-born youth, and those youth who were new to the Adelante program (67\%) showed increases in Confidence. For future Photovoice programs with youth who are below the age of fourteen, recent immigrants or new to the program, we recommend the use of an Optional Module 1: Team Building and Self-Empowerment prior to the Photovoice sessions to prepare these youth for full participation and to familiarize them with their facilitators and peers. The use of this optional module will prepare participants to fully engage in the Photovoice sessions as intended. The focus of this 
module is to build rapport with peers, build confidence in expressing ideas in front of a group, and the importance of working together with other youth to voice opinions.

\section{Disengagement from Community Issues}

Participants also differed in their level of familiarity with and engagement in community issues at baseline. The goal of the Photovoice program is to engage youth in community issues, but this was challenging with youth who were very disengaged from their community at baseline. Photovoice discussions were richer among participants who had a greater awareness of community issues and Competence for Civic Action at baseline. U.S.-born and foreign-born youth had similar levels of Civic Action Competence at baseline; however, 83\% of foreign-born youth showed improvement in this competence post-program, whereas U.S.-born youth did not. For those participants who are disconnected from community issues at baseline (due to lack of awareness or recent arrival in the community), we recommend using Optional Module 2: Community Activism to enhance participants' awareness of their community environment and explore the importance of youth voice in social change.

\section{Length of Time in U.S.}

Despite showing improvements in Civic Action Competence, the majority of Photovoice participants who had arrived to the U.S. more recently showed less or no improvement in Confidence, Connection to Peers, and Connection to Community. We recommend using Optional Module 3: Transitioning to the U.S. prior to Photovoice sessions to connect these youth with participants who have lived in the U.S. for longer periods of time, who can be understood as "Youth Ambassadors." The Youth Ambassadors could serve as peer mentors, help new immigrants familiarize themselves with their new community, and could help recently arrived youth feel more comfortable integrating into the Photovoice group. This also provides an opportunity for both recently-arrived youth and youth who have been in the U.S. longer to reflect on their experiences in transitioning to the U.S. and how their life in the U.S. may be similar or different to life in their home country.

\section{Figure 4}

Recommended Photovoice Curriculum for Immigrant Latino Youth

Optional Module 1: Team-building and Self-empowerment

Optional Module 2: Community activism

Optional Module 3: Transitioning to the US

Session 1: Introduction to Photovoice

- Photography: Camera Basics and Elements of a Photograph

- Ethics in Photography

Photo assignment \#1 - Portrait, Landscape, Macro

Session 2: Photo Review/Discussion, Recap of Composition/Technique \& Field Trip

- Assignment \#1 Review \& Discussion

Photo assignment \#2-What is it like to be a youth in your community

- Brainstorm session about what is it like to be a youth in your community - talk about potential ideas to be represented through photography.

- Field Trip to Photography Exhibit (professional or with socially-relevant theme)

Session 3: Photo Review/Discussion, Guest Speaker \& Photo Expedition

- Assignment \#2 Review \& Discussion 
- Guest Speaker sharing past Photovoice project, professional photographer, or community activist

Photo Assignment \#3-What in my community helps me to be healthy, active and eat well? What in my community prevents me from being healthy, active and eating well?

- Brainstorm session about what in my community helps us to be healthy, active and eat well - talk about potential ideas to be represented through photography.

- Community Photography Expedition

Session 4: Photo Review/Discussion \& Photography and Social/Policy Impact

- Assignment \#3 Review \& Discussion

- Discussion of How Photography Can Achieve Social/Policy Change

Photo Assignment \#4- What do you like/dislike about your community?

- Brainstorm session about what you like/dislike in your community - talk about potential ideas to be represented through photography.

Session 5: Photo Review/Discussion \& Exhibit Preparation

- Assignment \#4 Review \& Discussion

- Photo Exhibit Preparation Activity - discussion of invitees, materials needed, format

- Selection of Photos/Creation of Captions for Exhibit

Session 6: Preparation for Exhibit

- Creation/sending of invitations for community stakeholders, attendees

- Printing and framing of photos

- Finalization of logistics and materials for exhibit

Session 7: Online Exhibit and Advocacy

- Overview of online advocacy

- Engaging in online discussions around community issues portrayed in the exhibit

- Ethical considerations for online communications

\section{Limitations}

There were some limitations to the Photovoice pilot project that should be noted. We did not measure changes related to leadership, photography skills, empowerment, or verbal communications skills, which would have contributed to findings. Furthermore, although the sample size of participants is similar to other Photovoice projects, in contrast to other Photovoice projects, we also present quantitative pre-post measures of PYD assets. The small sample size introduces limitations in interpreting these findings.

\section{Discussion}

Similar to other Photovoice projects with Latino youth, this Photovoice pilot program showed promise in working with both recently arrived and first generation Latino immigrant youth to engage them in community issues and enhance their abilities to advocate for change that is important to them. We have additional recommendations regarding the utility of this curriculum in working with this youth population, as well as recommendations that may facilitate successful implementation of similar programs. 


\section{Photovoice as a PYD Intervention}

The program shows promise as a tool for development of PYD assets among Latino immigrant youth, and should continue to be tested with this population using the revised curriculum and added optional modules. As noted in the results, improvements were seen among at least half of all participants for the PYD assets of Confidence, Connection to Peers, Connection to Community, and two-thirds of participants showed increases in Competence for Civic Action, with the most improvement being seen among U.S.-born youth who were previous participants in Adelante program activities. This Photovoice program should be tested in other communitybased PYD interventions that seek to engage youth in community development, program planning and also improve PYD assets.

\section{Integrating Photovoice into Existing Programs}

This Photovoice curriculum can be integrated into existing youth programs or be a stand-alone program. Results from this pilot study suggest that participants who had previous involvement with the Adelante program tended to show more improvement in PYD assets than those youth who had never participated. This argues for the inclusion of Photovoice as part of a larger ongoing youth program. When implemented as part of an existing program, the Photovoice assignments can be tailored to emphasize a particular issue, delve deeper into a topic, assess the community environment surrounding an issue, and explore behaviors in more depth. This serves as an additional tool for facilitators, especially when a particular program has the goal of eliciting youth perspective and disseminating this perspective to a broader audience. When using Photovoice as a stand-alone curriculum, the photo assignments should center on some theme of interest to youth, or the assignments may be broader, such as in a community needs assessment.

\section{Photovoice for Participatory Intervention Planning}

This Photovoice pilot program served the dual purpose of building PYD assets among youth and also informing researchers and program implementers about evolving community needs from a youth perspective. In this way, youth have played a role in guiding future Adelante activities. Adelante youth and parent leader groups have joined forces with community stakeholders to address the issue of inadequate trash collection in the community, and findings from the Photovoice project have also informed efforts to address issues of inadequate nutrition and obesity in Langley Park. Future projects should consider the use of Photovoice to inform ongoing interventions about needs that may evolve in dynamic immigrant community contexts. We plan to use the revised Photovoice curriculum periodically with Adelante youth in order to include youth in the planning of the program as well as to stay in touch with evolving community needs.

\section{The SHOWeD Method}

The SHOWeD method for facilitating discussions demonstrated varying levels of effectiveness for this population. Among participants who were more willing to express their opinions, the SHOWeD method was adequate for eliciting responses. For younger, more recently arrived or youth who were new to the program, richer discussions emerged after occasional probing and follow-up questions. In some cases, considerable deviation from the SHOWeD method was required to elicit responses. The Optional Modules may be used to prepare youth for the Photovoice process, or future programs should consider adding probing questions to facilitate discussion with similar populations.

\section{Age of Participants}

Consideration should be given to the age of participants when planning a Photovoice program. All youth demonstrated exceptional capacity to use the cameras and take well-composed 
photos. However, the youngest participants had more difficulty in explaining their photos, relating the photos to broader community issues, and creating photo captions. If Photovoice youth are under age 14, facilitators should plan a more structured approach, making use of guided photo expeditions and structured discussions. We recommend recruiting participants from a more narrow age range since some younger participants seemed hesitant to speak out in a group that also included older participants.

\section{Unsafe Community Context/Photo Expeditions}

It was common among some youth to take photos exclusively in and around their homes, possibly also related to an unsafe community context and limitations in accessing other parts of the community. We share the recommendation of Strack, et al. (2004) for the use of guided photo expeditions, where youth decide community locations to visit, and are accompanied there by adult facilitators. These expeditions provide an opportunity for facilitators to deliver hands-on instruction on photo composition, camera operation, and requests for photo releases, as well as provides an opportunity for more varied photo content and team building through shared experiences.

\section{Facilitator Characteristics}

One key consideration for future programs should be the characteristics of the facilitators. In this program, all facilitators were bilingual, bicultural or very familiar with Latino culture and the immigrant experience. Facilitators who led sessions had a friendly, engaging, warm demeanor that made most youth feel comfortable in expressing their opinions. Since many of the youth were bicultural or had recently arrived to the U.S., having facilitators who could "operate" in both cultures and languages allowed youth to express themselves in the way that was most comfortable to them. Future programs should have facilitators who are trained in working with immigrant youth and have familiarity with both U.S. and home country cultures and contexts.

\section{Online Photovoice Exhibits and Use of Social Media}

Particularly important when working with youth, an online or social media component adds value to Photovoice programs. This mechanism increases reach in raising awareness among a broader audience of youth and community stakeholders. The Photovoice exhibit is available on the Adelante program's website, and there are plans to use the photos as advocacy tools and an engagement strategy for program youth. We did not make extensive use of social media, such as Facebook or Instagram in this pilot program, but plan to do so and recommend other Photovoice programs with youth to do the same.

\section{Conclusions}

Photovoice as a participatory method has become increasingly popular, and has been used to address a number of health and social issues. It is a useful tool for giving a voice to previously unheard community members and informing policy (Strack, et al., 2004; Wilson, et al., 2007). Photovoice has demonstrated considerable potential for engaging and empowering marginalized communities, especially immigrant youth. It has been less commonly used as a PYD strategy, but this pilot program shows that the Photovoice methodology can also contribute to the positive development of youth across a number of assets. Most importantly, youth participants are reassured that their perspective counts. Being involved in the Photovoice experience contributes to identity formation of youth, builds a sense of self-worth, and instills a greater understanding of where they fit into the world around them. 
In order to be successful, Photovoice programs with immigrant Latino youth need to take into consideration their unique circumstances, including length of time living in the U.S., level of comfort with self-expression, level of engagement in local community issues, age, and potentially unsafe community contexts. The Photovoice curriculum developed by our team takes in to account many of these issues and should continue to be tested with other youth. The curriculum is brief, the program is relatively inexpensive to implement, can be easily adapted to meet the needs of participants, and can be integrated into existing programs. This curriculum may be particularly relevant for public health practitioners, health educators and youth program implementers. Immigrant youth deserve a mechanism through which they can participate in dialogue about community issues. Photovoice is a promising approach for facilitating youth engagement and empowerment, and with a curriculum that meets the unique circumstances and needs of immigrant youth, this can be better achieved.

\section{References}

Agency for Healthcare Research and Quality. (2009). Retrieved from

http://www.ahrq.gov/research/findings/factsheets/primary/cbprbrief/index.html

Cleary, S.D. (2014). Washington, DC:

Edberg, M., Cleary, S., Collins, E., Harris, M., McLendon, H., \& Santucci, P. (2009). Patterns of HIV/AIDS, STI, substance abuse and hepatitis risk among selected samples of latino and african-american youth in washington, DC. Journal of Youth Studies, 12(6), 685-709.

Erikson, E.H. (1968). Identity: Youth and crisis. New York: Norton.

Freire, P. (1973). Education for critical consciousness. New York: Continuum.

Garcia, C.M., Aguilera-Guzman, R.M., Lindgren, S., Gutierrez, R., Raniolo, B., Genis, T., Clausen, L. (2013). Intergenerational photovoice projects: Optimizing this mechanism for influencing health promotion policies and strengthening relationships. Health Promotion Practice, 14(5), 695-705.

Greene, S., Burke, K., \& McKenna, M. (2013). Forms of voice: Exploring the empowerment of youth at the intersection of art and action. Urban Review, 45(3), 311-334.

Hergenrather, K.C., Rhodes, S.D., Cowan, C.A., Bardhoshi, G., \& Pula, S. (2009). Photovoice as community-based participatory research: A qualitative review. American Journal of Health Behavior, 33(6), 686-698.

Israel, B.A., Eng, E., Schulz, A.J., \& Parker, E.A. (2005). Methods in community-based participatory research methods for health. San Francisco: Jossey-Bass.

Killion, C.M., \& Wang, C.C. (2000). Linking African American mothers across life stage and station through photovoice. Journal of Health Care for the Poor and Underserved, 11(3), 310325. 
Lerner, R.M. (2005). Promoting positive youth development: Theoretical and empirical bases. $<\mathrm{br} />$ (Workshop on the Science of Adolescent Health and Development Ed.). Washington, DC: National Research Council.

Martinez Jr., C.R. (2006). Effects of differential family acculturation on latino adolescent substance use. Family Relations, 55(3), 306-317. doi:10.1111/j.1741-3729.2006.00404.x

Martinez, C.R., Eddy, J.M., \& DeGarmo, D.S. (2003). Preventing substance use among latino youth. In W.K. Bukoski, \& Z. Sloboda (Eds.), Handbook of drug abuse prevention: Theory, science and practice (). New York: Kluwer Academic/Plenum.

Minkler, M., \& Wallerstein, N. (2003). Introduction to community based participatory research. In M. Minkler, \& N. Wallerstein (Eds.). Community based participatory research for health (pp. 3-26). San Francisco: Jossey Bass.

Morales-Campos, D.Y., Parra-Medina, D., \& Esparza, L.A. (2015). Picture this! Using participatory photo mapping with hispanic girls. Family and Community Health, 38(1).

Rhodes, S.D., Hergenrather, K.C., Griffith, D.M., Yee, L.J., Zometa, C.S., Montaño, J., \& Vissman, A. (2009). Sexual and alcohol risk behaviours of immigrant latino men in the southeastern USA. Culture, Health and Sexuality, 11(1), 17-34.

Roffman, J.G., Suarez-Orozco, C., \& Rhodes, J.E. (2003). Facilitating positive development in immigrant youth: The role of mentors and community organizations. In F.A. Villaruel, D.F. Perkins, L.M. Borden \& J.G. Keith (Eds.), Community youth development: Programs, policies and practices (pp. 90-117).

Schwartz, L R., Sable, M.R., Dannerbeck, A., \& Campbell, J.D. (2007). Using photovoice to improve family planning services for immigrant hispanics. Journal of Health Care for the Poor and Underserved, 18(4), 757-766.

Silbereisen, R.K., \& Lerner, R.M. (2007). Approaches to positive youth development. London: Sage Publications.

Stevens, C.A. (2010). Lessons from the field: Using photovoice with an ethnically diverse population in a HOPE VI evaluation. Family and Community Health, 33(4), 275-284.

Strack, R.W., Magill, C., \& McDonagh, K. (2004). Engaging youth through photovoice. Health Promotion Practice, 5(1), 49-58.

Streng, J.M., Rhodes, S.D., Ayala, G. X., Eng, E., Arceo, R., \& Phipps, S. (2004). Realidad latina: Latino adolescents, their school, and a university use photovoice to examine and address the influence of immigration. Journal of Interprofessional Care, 18(4), 403-415.

US Census Bureau. (2012).

Vaughn, L.M., Rojas-Guyler, L., \& Howell, B. (2008). "Picturing" health: A photovoice pilot of latina girls' perceptions of health. Family and Community Health, 31(4), 305-316. 
Vaughn, S. (2012). Stroke and heart disease prevention education via telenovela: A focus group's evaluation. Rehabilitation Nursing, 3ð5) doi:10.1002/rnj.053.

Vega, W.A., \& Gil, A.G. (1998). A model for explaining drug use behavior among hispanic adolescents. Drugs and Society, 14(1-2), 57-74.

Wang, C., \& Burris, M.A. (1997). Photovoice: Concept, methodology, and use for participatory needs assessment. Health Education and Behavior, 24(3), 369-387.

Wang, C., Morrel-Samuels, S., Hutchison, P.M., Bell, L., \& Pestronk, R.M. (2004). Flint photovoice: Community building among youths, adults, and policymakers. American Journal of Public Health, 94(6), 911-913.

Wilson, N., Dasho, S., Martin, A.C., Wallerstein, N., Wang, C.C., \& Minkler, M. (2007). Engaging young adolescents in social action through photovoice: The youth empowerment strategies (YES!) project. Journal of Early Adolescence, 2オ2), 241-261. doi:10.1177/0272431606294834.

Youniss, J., \& Yates, M. (1997). Community service and social responsibility in youth. Chicago: University of Chicago Press.

(C) Copyright of Journal of Youth Development Bridging Research and Practice. Content may not be copied or emailed to multiple sites or posted to a listserv without copyright holder's express written permission. Contact Editor at: patricia.dawson@oregonstate.edu for details. However, users may print, download or email articles for individual use.

ISSN 2325-4009 (Print); ISSN 2325-4017 (Online) 